\title{
Multiscale Modeling of Cellular Systems in Biology
}

\author{
J. C. Dallon \\ dallon@math.byu.edu
}

Follow this and additional works at: https://scholarsarchive.byu.edu/facpub

Part of the Mathematics Commons

\section{Original Publication Citation}

Current Opinion in Colloid and Interface Science15(1-2): 24-31

\section{BYU ScholarsArchive Citation}

Dallon, J. C., "Multiscale Modeling of Cellular Systems in Biology" (2010). Faculty Publications. 2713. https://scholarsarchive.byu.edu/facpub/2713 


\title{
Multiscale Modeling of Cellular Systems in Biology
}

\author{
John C. Dallon \\ Department of Mathematics \\ Brigham Young University \\ $374 T M C B$ \\ Provo, UT 84602-6539 \\ U.S.A.
}

\begin{abstract}
Here we review eight different multiscale modeling efforts dealing with cellular systems in biology. The first two models focus on collagen based tissue, one dealing with the biomechanical properties of the tissue and the other focusing on how the dermis is remodeled in scar tissue formation. The next two models deal with first avascular tumor growth and then the role of the vasculature in tumor growth. We then consider two models which use the Immersed Boundary method to model tissue properties and cell-cell adhesion. Finally we conclude with two models with treatments of the Cellular Potts Model. The first models somitogenisis in the chick and the second links the Cellular Potts Model with the Keller-Segel model.
\end{abstract}

\section{Introduction}

The life sciences are a rich field well suited for the application of multiscale modeling. Multiscale modeling applies to modeling where different space, time, or other variable scales are inherent in the problem. When developing a model, careful consideration of the different scales is necessary to accurately model the system. In biology there are naturally many space and times scales which often are mixed in the same system. Living systems have space scales which can range from kilometers, the habitat of an organism, to microns or less when considering phenomena at the cellular level. Even when dealing with a single organism, the space scales can range from meters to microns. Internal to a cell, the space varies from microns to nanometers. The time scales are equally varied. Measurements of population growth can be on time scales from years to hours. Cell division occurs on a scale of hours but is determined by processes with time scales of seconds. Ignoring the different scales can lead to erroneous conclusions or make the problem computationally untractable.

Email addresses: dallon@math.byu.edu (John C. Dallon) 
Although there are several examples in population dynamics, ecological qmodeling, migratory patterns, and others, this review will focus on cellular systems. First we consider two models of collagen based tissues. The first model by Baracos and colleagues [27] considers individual fiber forces in a volume averaging way to determine the biomechanical properties of collagen tissue. The second model by Dallon and co-workers [15] considers the biochemical remodeling of the collagen in the dermis in scar tissue formation. Next we review two models of tumor growth, one model by Anderson et. al. which models avascular tumors with cellular automata [3] and one by Brynes and co-authors [18] which deals with vascular tumorous tissue and how the remodeling of the vasculature can affect the tumor growth. We then review two models which use the Immersed Boundary method. The first model focuses on tissue properties and is applied to tumor growth, the structure of trophoblast tissue in the placenta, and the development of epithelial acini [22]. In the second model using the Immersed Boundary method, the role of cadherin motion within the cell membrane is explored in the context of cell-cell adhesion [9]. Finally, we conclude with two models dealing with the Cellular Potts Model. The first work models somitogenesis in the chick [11] and the second connects the Cellular Potts Model with the Keller-Segel model [2]. There are two recent books containing a compilations of multiscale models in developmental systems [25]* and cell-based models $[4]^{*}$ which may be of particular interest.

\section{Models of fibrous collagen networks}

In the area of tissue engineering, Barocas and co-workers have modeled collagen using multiscale methods and Dallon and colleagues have used multiscale models to understand collagen alignment in the dermis. Collagen is the main connective protein in the human body. It polymerizes into fibrils, small fibers, which join together forming larger collagen fibers. The organization and structure of the fibrous network determines the mechanical properties of the tissue. In tendons, the collagen fibrous network is highly aligned with large dense collagen fibers. This gives the tendons strength to resist forces in one direction. In the dermis, the collagen network is not as dense as in tendons and the collagen aligns in a cross weave pattern which is anisotropic. That is, the cross weave pattern does not persist in any particular direction. On a small scale the collagen aligns in two directions, but at another nearby location the directions of alignment are typically different. This gives the dermis strength to resist forces in any direction. In scar tissue the alignment and structure of the collagen network is different, resulting in a weaker and less functional region in the dermis. In the cornea the collagen is organized in orthoganal sheets of fibrils, somewhat like the structure of plywood. The size and organization of the collagen fibrils determines the mechanical and optical properties of the cornea.

Because collagen forms a fibrous network and the structure of the network determines the properties of the tissue, multiscale modeling is a natural technique to use when modeling the mechanical properties of collagen. The microstructure of the collagen network which is on the scale of microns determines the 


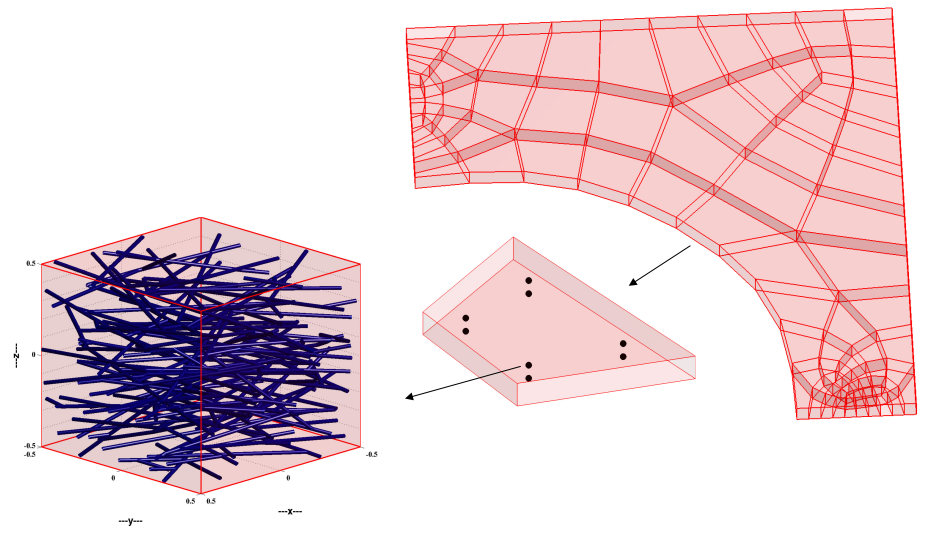

Figure 1: The figure shows a piece of tissue being modeled and the different scales of the problem. The Upper right shows the piece of tissue subdivided into the finite elements used in the Galerkin method. In the middle one of the elements is shown with the integration points. In the lower left, the RVE centered at the integration point is shown with its collagen structure (courtesy of E. A. Sander and V. H. Barocas [24]).

properties of the tissue, where the space scale is of the order of centimeters. Typically the tissue properties are of greatest interest, but to understand them requires understanding the microstructure of the network and the interactions at the scale of the fibers.

\subsection{Volume averaging}

One method for modeling collagen tissue properties is volume averaging. In this method the domain is subdivided into representative volume elements (RVE). The RVE are modeled in some manner to give an average response for the RVE. The average response is then used in a macroscopic model over the entire domain. Barocas and colleagues have applied this approach to general collagen networks $[27,14,7]$ and to the elastic properties of the arterial wall [26]. A novel aspect of their work is that the fibrous structure of the collagen is modeled at the level of the RVE (see figure 1). From that submodel the average strain-induced stress is computed and used to compute the deformation of the tissue as a whole.

Barocas and co-workers model collagen tissue in the following manner. They divide the collagen network into subregions (finite elements), which are divided further into RVEs. Each RVE is initially populated with fibers by randomly 
locating nucleation sites in the RVE. The fibers grow in opposite directions from the nucleation site, the direction is randomly chosen, until the fiber leaves the RVE or collides with another fiber. If fibers collide a cross link is formed and the fiber stops growing. Both random anisotropic fibrous structures as well as preferentially aligned networks can be modeled.

The force on the individual fibers is modeled by a nonlinear equation given by

$$
f=\frac{E_{f} A_{f}}{B}\left(e^{B \epsilon}-1\right)
$$

where $\epsilon$ depends on the fiber's stretch ratio. The average stress for the RVE, $S_{i j}^{S}$ is calculated by

$$
S_{i j}^{S}=\frac{\eta}{a} \sum x_{j} f_{i} .
$$

The sum is over the boundary cross-links and $x_{j}$ is the location of the cross-link. The force balance for the macroscopic scale is given by

$$
S_{i j, i}=\frac{1}{V} \oint\left(s_{i j}-S_{i j}\right) u_{k, i} \eta_{k} d S,
$$

where ", $i$ " is differentiation with respect to $x_{i}, u$ is the displacement of the RVE boundary, $\eta_{k}$ is the unit normal vector, and $s_{i j}$ is the microscopic stress tensor. The macroscopic problem is solved using a Galerkin finite element method. The nonlinear problems are solved using a Newton-Raphson iteration. To give an idea of the computational resource necessary for this type of modeling, in one problem eight RVE's per finite element were used. Each RVE had over 300 fibers [26]. The computational time is dominated by the microscopic fiber computations take most of the time.

This model framework has successfully modeled several situations. It has modeled the deformation of simple collagen slabs [27] matching the experimental stress-strain curves nicely. The model was extended to model the effect of water in the collagen network [7]. In this work the hydrated fiber networks are modeled at the macroscopic level with two-phase equations. The two-phase equations are derived from volume averaging. The mechanical phase is similar to what has been described. The fluid phase is modeled as an incompressible Newtonian fluid which has a drag effect on the fibers based on Stokes flow around a cylinder and the macroscopic fluid velocity is determined using Darcy's law. The model shows that the larger the rate of deformation, the resistance of the fluid phase to compression becomes more significant, but the equilibrium state remains the same. The volume averaging of collagen has also been applied to the arterial wall where there is a complicated geometry and the tissue has a more complex structure $[26,14]$. The arterial wall is composed of three layers: the innermost layer is composed of endothelial cells and a basal membrane, a middle layer of collagen, smooth muscle cells and elastin fibers, and the outer layer of collagen, fibroblasts, elastin, and proteoglycans. The model considers only the collagen and the noncollagen matrix. The collagen is modeled as previously described. The noncollagen matrix is a compressible neo-Hookean material treated as a 
continuum. The collagen and the noncollagen matrices are independent and their stresses add. The collagen in the middle layer wraps around the artery. Thus the RVE have the fibers preferentially aligned to mimic this. The model matched experimental values for pressure and wall thickness. The model further predicts that the noncollagen matrix not only resists deformations but also helps to preserve the shape of the tissue.

\subsection{Dermal Wound Healing}

Multiscale modeling has been applied to collagen structures in dermal wound healing by Dallon and co-workers [15, 10]. Their treatment of the collagen matrix is very different from the previous work discussed. The multiple scales of the problem are similar to those previously described, there is the molecular scale with diffusing cytokines, the subcellular level with production of proteins and cytokines within the cells, the scale of the collagen fibers, the cellular scale, and the tissue scale.

This modeling focuses on wound healing in the dermis. The dermis is primarily composed of collagen in the form of a fibrous structure. There are relatively few cells in normal dermal tissue. When the dermis is wounded there is a complex process which is initiated to replace the damaged tissue. The work described here deals with the tissue regeneration phase of wound healing. In this phase, fibroblasts, cells responsible for the production of connective tissue, enter the wound region which is composed of a provisional matrix produced by the blood clot. The fibroblasts replace the provisional matrix, composed primarily of fibrin, with new dermis or a matrix compose primarily of collagen. When scarring occurs the new dermis has a different structure than the original dermis. The modeling by Dallon et. al. is primarily concerned with the orientation of the collagen structure. In normal tissue the orientation is a cross weave pattern and in scar the orientation is different.

The model treats the extracellular matrix as a continua, the cells as discrete objects, and the diffusable substances as continua. The extracellular matrix is represented by two variables, one which denotes the density of collagen and the predominant orientation of the collagen and another variable which denotes the same quantities for fibrin, the main component in the provisional matrix. The cells and the extracellular matrix interact in two main ways. The first is by contact guidance. The fibrous matrix guides and determines how the cells migrate in the matrix. The second interaction is the cells degrade the provisional matrix and replace it with collagen. In this manner the cells invade the provisional matrix and replace it with new dermis.

In the model the subcellular processes are modeled with ordinary differential equations (ODEs). These ODEs determine the degradation and production of the extracellular matrix local to the cell location. The extracellular matrix is discretized for the computational simulations. Once it is discretized it can be thought of as volume averaging with the discrete function representing the average values of the extracellular matrix at that location. The cells move according to the density of the extracellular matrix and in the direction determined by the collagen and fibrin structures. The cells are also chemotactically guided by a 
diffusable substance which is produced in the wound region, diffuses and is degraded. The diffusable substance is modeled with a partial differential equation (PDE).

The authors learn many things from the model. They find, in a simplified version of the model with only collagen as the extracelluar matrix, that how the cells enter the domain and how the initial extracellar matrix is aligned are important factors in determining the overall alignment of the remodeled matrix. When fibrin is added to the model as the provisional matrix which is replaced with a collagen matrix, the random nature of the fibrin plays a dominant role in determining the final alignment patterns. For instance, as the cells enter the provisional matrix their direction is dictated by the random fibrin matrix. This makes it hard for them to penetrate into the wound region and causes a boundary layer to form. This boundary layer can be overcome by adding a diffusable chemoattractant which is produced in the wound region. It can counter the randomizing effect of the provisional matrix, help direct the cells into the wound region and prevent a boundary layer from forming. If the effect of the chemoattractant is strong, it can cause significant alignment due to its directional effect on the cells. Thus a careful balance between directing the cells into the region and not causing alignment would be necessary for scarless wound healing. Finally, the authors conclude that an agent which causes the fibroblasts to sample their environment more frequently, so the cells can change directions more often, would be an effective antiscaring agent.

\section{Multiscale Model of Tumor Growth and Invasion}

\subsection{Avascular Tumor Growth}

There have been several mathematical models of tumor formation and growth [6]. A solid tumor is typically initiated when a mutated cell or cells are able to circumvent the normal regulatory procedures in the body which control cell growth. These cells start to proliferate, crowding out normal cells. The tumor grows avascularly, without blood cells, due to nutrient and waste diffusion. When the tumor reaches a particular size, the constraints due to the nutrient and waste diffusion inhibit growth. For further development of the tumor, vascularization is needed. Some tumors at this stage can recruit the growth of blood vessels which not only alleviates the growth constraints but allows some cells to break away from the initial tumor and travel to other locations forming secondary tumors. As the tumor grows, more mutations can occur, resulting in a heterogeneous tumor.

Anderson and co-workers [3] are working on models of tumor growth and invasion using multiscale modeling techniques. In the work we review here, they model events at the genetic, subcellular, cellular, and tissue level. The authors start with a system of four differential equations representing tumor cell density $n$, matrix degradative enzymes $m$, the extracellular matrix molecules $f$, and the oxygen concentration $c$. The differential equations are:

$$
\frac{\partial n}{\partial t}=D_{n} \nabla^{2} n-\chi \nabla \cdot(n \nabla f),
$$




$$
\begin{aligned}
\frac{d f}{d t} & =-\delta m f, \\
\frac{\partial m}{\partial t} & =D_{m} \nabla^{2} m+\mu n-\lambda m, \\
\frac{\partial c}{\partial t} & =D_{c} \nabla^{2} c+\beta f-\gamma n-\alpha c .
\end{aligned}
$$

These equations, which have continuous variables, are then discretized. The discretization has two purposes. First, it allows the equations to be solved numerically, and second, it allows the variable for the cell density $n$ to be treated as individual cells. In this formulation, all the variables except $n$ are thought of as continuous variables. They are concentrations of molecules and have a much smaller scale than the cells (the subcellular scale).

The cells can now be treated as individuals or averages of individuals with distinct properties (the cellular scale). This allows for stochastic cell migration, adhesion, proliferation, necrosis, and cell mutation. A cell is allowed to either stay at its current location, move south, west, north, or east to adjacent grid points. The discretized equation for the cells is modified so that stochastic cell motion can be incorporated by prescribing probabilities for each of the possible motions. The probability of motion is determined by the concentration of the degraded extracellular matrix $f$, to account for haptotaxis, and also depends on local cell density to account for cell-cell adhesion. Cells will die if the oxygen concentration is too low and they will proliferate if there is sufficient space for the new cell. Different cell phenotypes have different properties of adhesion, oxygen uptake, cell motion, and matrix degradation.

Cells can change phenotypes due to random mutations. This is treated in two manners depending on the simulation. One is the linear algorithm where the cells all start as one phenotype, type I, and can mutate with a small probability into, type II. Type II cells can mutate into type III cells, which can mutate into type IV cells. There are only four phenotypes. Each one is supposed to be a more aggressive type of cancer. The mutations are irreversible. The other algorithm for mutation is random. In this algorithm, there are 100 phenotypes with randomly chosen properties for proliferation age, oxygen consumption, extracellular matrix degradation properties, and motion properties. These mutations are reversible and a cell can mutate into any one of the 100 phenotypes. The initial cell population may or may not be homogeneous with respect to the phenotypes in this algorithm.

Anderson and colleagues found both algorithms for genetic mutation gave similar macroscopic patterns for tumor growth. When the extracellar matrix molecules were initially set at a constant level, both algorithms resulted in a compact round tumor shape. When the initial extracellular matrix is nonhomogeneous with either a random intial distribution or complex pattern, both mutation algorithms produced tumors with a fingered morphology. In addition they found in the uniform extracellular matrix simulations there were more competing phenotypes of cells using the random algorithm than when the extracellular matrix was non-homogeneous. They concluded that the extracellular 
matrix is very important in determining the morphology of the tumor and in selecting for the more aggressive tumor phenotypes.

They additionally tested the effects of altering the oxygen concentration. Experiments have shown that tumor spheroids grown in a normal oxygen environment remain spherical and compact, whereas spheroids grown in oxygen deprived (hypoxic) conditions have a fingered morphology [19]. The mathematical model was able to reproduce the same type of results. The model also found that in the normal oxygen environment there were many competing phenotypes of cells. In the oxygen deprived environment only a few aggressive cell types remained.

\subsection{Vascular Tumor growth}

Unlike the previous models which deal with avascular tumor growth, the work of Byrne and co-authors focuses on the effect of adding vasculature and the process of angiogenesis, the formation of blood vessels, to tumor growth $[18,1]$. The time scales in this problem range from scales in blood flow, the time it takes for the vascular network to change, changes in oxygen levels and cytokine levels in the tissue, changes in the cancerous cells - growth and death. The space scales are also varied including those from the molecular level, cellular level and the vasculature network level. The authors consider the vascular layer, the cellular layer, and the subcellular layer. The vascular layer and the cellular layer are coupled through diffusive transport of oxygen and VEGF, a cytokine which stimulates angiogenic sprouting.

The vascular layer of the model consists of an initial hexagonal network of blood vessels which can mature. The radius of the blood vessels can increase or decrease depending on several factors including VEGF, blood flow rate, and the haematocrit, which is the fractional volume of red blood cells to blood volume. The equation governing the radius of the blood vessels is

$$
\begin{aligned}
R\left(t+\epsilon_{t}\right) & =R(t)+\epsilon_{t} R(t)\left(S_{h}+S_{m}+S_{d}+S_{u}-k_{s}\right) \\
S_{h} & =\log \left(\tau_{w}+\tau_{r e f}\right)-k_{p} \log (\tau(P)) \\
S_{m} & =k_{m}(V) \log \left(\frac{\dot{Q}_{r e f}}{\dot{Q} H}+1\right),
\end{aligned}
$$

where $S_{h}$ is the haemodynamic stimulus, $S_{m}$ is the metabolic simulus, $k_{s}$ is the shrinking stimulus, $\dot{Q}$ is the flow rate assumed to be a laminar steady Poiseuille flow, and $H$ is the haematocrit.

Angiogenesis or new blood vessel formation is included by modeling capillary sprouts. The sprouts grow by having the tips perform a random walking on a lattice with a bias towards high levels of VEGF. When the capillary tip joins with another tip or another blood vessel, in the process of anastomosis, the sprout is added to the vascular network and its radius can change. If the tips do not join other tips in a certain time frame, the tip will die or regress. In this manner the role of angiogenesis and vessel pruning can be modeled and its effects determined. 
The cellular layer is modeled by a cellular automaton. The cell-cell interactions between normal and cancerous cells are considered. Cell death or apoptosis, division, and crowding are considered. Cell behavior is determined by the diffusable elements which are oxygen, VEGF, and p53, which regulates apoptosis.

The subcellular layer is modeled with a system of seven ODEs for each cell in the cellular automaton model. Five model the cell cycle and are an extension of the Tyson-Novak model [28]. The other two equations model a cell's production of p53 and VEGF and depend on the oxygen available to the cell.

Reaction diffusion equations model the transport of oxygen and VEGF through the tissue. The vasculature acts as a source for oxygen and a sink for VEGF with the strength depending on the vessel radius. The cells act as spatially-distributed sinks for oxygen and sources for VEGF.

The authors conclude from the model that vessel pruning is due to pressure drops across a vascular network. Tumor growth can have periods of stagnation followed by sudden growth. This is due to the time it takes for angiogenesis to form a major vessel which can supply the tumor with the necessary nutrients. Further, in tissue with a tumor the vasculature is more dense due to the greater demands for resources of the cancerous cells.

\section{Immersed Boundary Method}

In this section we review multiscale models of tissue and cell-cell adhesion which use the immersed boundary (IB) method as the basis for the model formulation. The IB method was first introduced by Peskin and was applied to modeling flow patterns around heart valves [20]. Since then it has been used to model many systems [21]. The IB method assumes a boundary surrounded by fluid which moves with the fluid (no slip boundary conditions) and can exert forces on the fluid. The fluid is assumed to be incompressible and the NavierStokes equations are used to solve the fluid velocity throughout the domain. The method assumes the same equations hold for the fluid over the entire domain with no boundary conditions at the IB. The boundary and the fluid interact with each other; the boundary alters the fluid velocity and the fluid velocity moves the boundary. The boundaries are typically elastic boundaries which apply Hookean forces to the fluid. There can be more than one IB and in the models described here the boundaries represent cell membrane and the fluid represents either the internal cytoplasm of the cell or the extracellular environment.

\subsection{Tissue modeling}

The work of Rejniak and co-workers [22, 5, 23] uses the IB method to model tumors, the tissue folds in placental trophoblast bilayer, and the development of stable acinar structures. The trophoblast tissue in the placenta plays a role in the barrier between the blood stream of the mother and the fetus. Epithelial acini are structures which are studied in hopes of better understanding acini in

the female breast. The female breast is formed by duct systems comprised of 
lobules which are terminal ductules and acini, or the alveolar structure of the breast. All of these tissues have been model using the IB method as a basis.

The model formulation for all these applications is the IB method. The same type of multiscale issues are found in these tissue problems as those described previously. There are subcellular processes, cellular processes, and the overall tissue scale. The cell membranes are treated as elastic boundaries immersed in an incompressible viscous fluid. The fluid represents the extracellular and intracellular regions. There is a domain which has several closed curves, the cell membranes. The model allows for cell growth and division, death, cellcell attachments, a response of a cell to respond to its microenvironment, and attachment to additional tissue elements like a basal membrane.

The cell growth and death require sinks and sources of the fluid thoughout the domain. In the IB method there should be no fluid flow through the boundaries (the numerical implementation will determine how much fluid leaks through). Of course, the area (for two dimensional simulations) of the domain is constant but the fraction of intercellular area to the total area changes as cells grow, divide, and die. The change is caused by the sources and sinks and represents the ability of a cell to allow fluid to pass through its cell membrane. When cells grow the intracellular area increases and when cells die, apoptosis, the intracellular area decreases. In the model, as a cell's area grows, the cell will form a contractile ring. The contractile ring is modeled by springs causing forces on opposite sides of the elastic membrane to bring it together. As the membrane is drawn close enough the cell will be split into two different cells. The process of apoptosis involves a loss of cell volume until the cell is removed from the simulation. Cell death also involves a loss of cell-cell attachments.

The cell-cell adhesion and adhesion to other tissue elements is modeled by springs from one membrane to another or a spring from a cell to a specified location in the domain. In the IB method the boundaries are described as a set of connected points. In cell-cell adhesion each point can be connected via a spring to a point on another cell, if the cells are close enough. If the cell is attached to some tissue component like a basal membrane then the spring attaches to the cell membrane and a fixed location in the domain which represents the tissue component.

Cells can respond to the microenvironment surrounding their membrane. The stimulus can be mechanical or chemical. For instance if the chemical concentration of a growth stimulus is high near a cell membrane, then the cell will grow by pumping extracellular fluid into its cell body.

Using this formulation the three types of tissues describe earlier have been modeled. In this formulation the biomechanical properties of the tissue can be investigated. In the tumor, both fingering type morphology and compact morphologies can be obtained. In the modeling of trophoblast, the model was used to invesitgate the abnormal folding of the tissue. In the trophoblast there are two cell layers CT (cytotrphoblast) and ST (syncytiotrophblast). The CT layer is tightly packed with cells which divided, whereas the ST layer can only grow by insertion of cells from the CT layer. In the model, the ST is modeled as one large cell with two boundaries representing the boundaries with other cells 

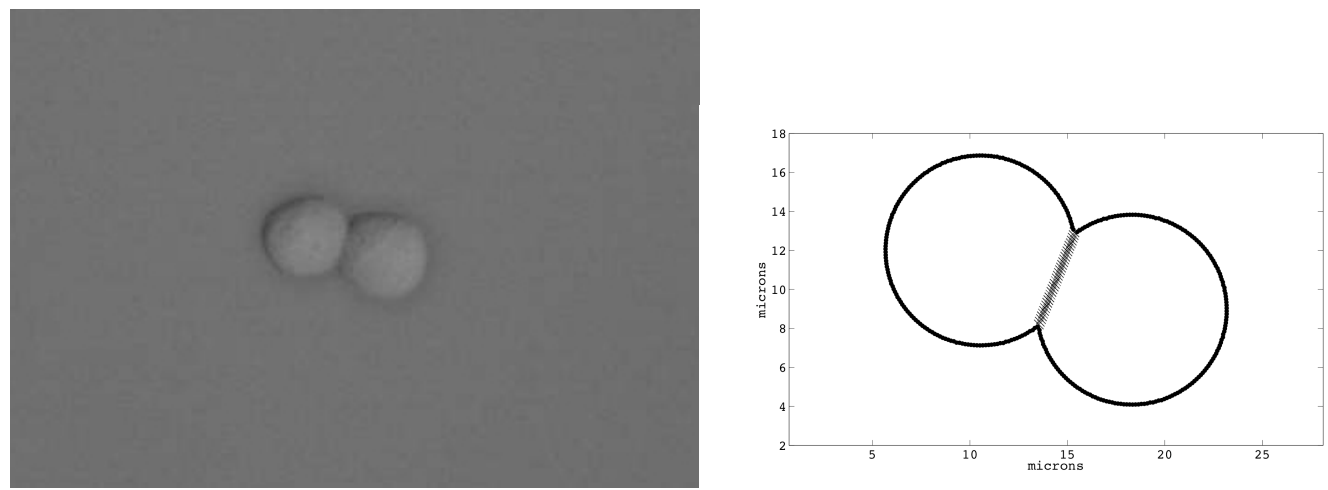

Figure 2: The panel on the left shows two Madin-Darby canine kidney (MDCK) cells in suspension culture (courtesy of M. D. H. Hansen). The panel on the right shows a typical simulation after 100 seconds where the cadherins are fixed in the cell membrane. The black dots represent 100 cadherin molecules and the $\mathrm{X}$ represents a bound cadherin.

outside the layer. In the CT layer, each cell is modeled as a IB. A network of vertical springs is used to model the cytoskeletal structure of all the cells and to maintain the shape of the two layers. The model can reproduce the different shapes of trophoblast tissue. The shapes that the tissue form are determined by the stiffness of the two layers. If the CT layer is too flexible, the cells vary significantly in shape. When both layers are moderately stiff, the tissue forms a flat morphology with no bending. If the CT layer is stiff but the ST layer is flexible, the CT layer can squeeze in an irregular manner into the ST layer. Finally, if both layers are stiff then the tissue will fold.

The final application is to the epithelial acini which are similar to structures in the female breast. In these simulations a single cell will grow into a cluster. The cells in the interior of the cluster will die and leave a hollow shell of cells or the acini. In the model as the cells divide the outer cells form tight adhesions with other outer cells and signal the inner cells to initiate apoptotic death. The process is complex and needs to be tightly regulated to form the correct structure in a stable manner.

\subsection{Cell-cell adhesion}

Another multiscale model which uses the IB method focuses on cell-cell adhesion [9]. In this work there are only two scales, the subcellular and the cellular. The purpose of the model is to explore how the influence of the actin cytoskeleton on the cadherins affects the cell-cell contact area (see figure 4.2).

In this model there are two IB, each one representing a cell membrane. The membranes are initially touching each other at one point. The model then simulates how the contact area between the two cells grows. The model is force based and examines how the forces affect the contact area between the cells. The forces involved are those of the elastic membrane, which are modeled as 
linear springs, those of the bulk fluid, and the forces of interacing cadherins, the transmembrane proteins which cause cell-cell adhesion.

In the model, cadherin complexes are located on the cell membrane and interact with cadherin complexes located on the other cell's membrane. When two cadherins from different cells get close enough they will interact with each other causing a force which is also modeled as a linear spring. Since cadherin complexes can diffuse in the cell membrane, the model explores how cadherin motion in the membrane affects the cell-cell contact area. The model allows for cadherin complexes fixed in the membrane, complexes which freely diffuse, and complexes which move due to convective forces. When two cadherins are interacting, the force due to the interaction and the membrane deformation can cause convective forces on the cadherin. By considering these forces the overall shape and configuration of the two cells can be determined.

The authors conclude that the final cell-cell contact area is largely independent of the diffusion coefficient for the cadherins, over a wide range of diffusion coefficients when only diffusive motion is considered. This indicates that, for cells in suspension, it is not necessary for a cell's cytoskeletal structure to anchor or restrict the motion of the cadherins in the membrane for the full cell-cell contact area to be achieved. It also indicates that the cadherins, once they are interacting with cadherins from another cell, do not need to be actively moved to achieve the full contact area. When cadherin convection is added to the model the conclusions remained the same. The cell-cell contact area plateaued sooner than in the case when the cadherins simply diffuse but the final area is roughly the same.

When the authors tried to crudely simulate adhesion to a substrate by increasing the viscosity of the surrounding fluid the contact length was drastically reduced. This suggests that pseudopodial activity or some other mechanism is required to achieve the observed cell-cell contact areas.

\section{The Cellular Potts Model}

The Cellular Potts Model (CPM) has been used to model several systems in developmental biology. Here we give a brief overview of the method and then discuss a specific application of the CPM to somitogenesis. The CPM is a generalization of the Ising model which is used to model magnetic interactions. In the Ising model there are discrete variables (spins) which can have one of two states. There are different energies associated when interacting variables have the same state or different states, and the overall energy of the system is minimized [12]. In the CPM, cells are described by the lattice sites which they occupy. Thus they can move and have complex shapes which change. The internal structure of the cell is not modeled. Cell motion and interaction is determined by an energy function which is minimized [8]. The energy func-

tion typically will have parts depending on adhesion between cells, volume of a cell, and concentration of a chemoattractant. The method is updated using a modified Metropolis Monte-Carlo algorithm. 
The left panel of figure 3 shows the system at one time. There are three cells in the domain represented by the three different colors (not including the white lattice region). In the Monte-Carlo algorithm, random lattice locations are chosen and updated according to how the energy of the system changes. For example, if the white lattice region is chosen for the black cell to occupy, a new energy for the system would be calculated. The volume of the black cell would increase, and usually there is a predetermined optimal volume for a cell. If the black cell is under the optimal volume, the energy of the system would decrease by adding more volume to the black cell so that its overall volume would be closer to the optimal volume. Additionally, the black cell would have more contact area with the two other cell types which may cause the energy of the system to increase (if the cells did not adhere well with other cells). Finally, if there was a chemoattractant causing the cell to move to the bottom of the figure, the energy of the system would decrease since the cell was moving in the correct direction. The overall energy would change in some manner by having the black cell occupy the white site. With some probability, the algorithm would accept the change depending on how the overall energy was affected. Lower energy states are accepted with a higher probability than higher energy states.

\subsection{Modeling Chick Somitogenesis}

The process of forming somites is called somitogenesis. As a vertebrate embryo develops, groups of cells separate into clusters called somites. These are precursors to dermis, skeletal muscle, and vertebrae. Glazier and others have modeled this process using the Potts model [11]. In their work they model subcellular events such as the expression of cadherins and other cellular proteins, individual cell migration, and the patterns at the tissue level of somite formation

In Glazier's model of somite formation, the authors assume a cell's mechanical activity is determined by different expression of cell-cell adhesion molecules $\mathrm{N}-\mathrm{CAM}$ and N-cadherin and by different expressions of EphA4 receptors and ephrinB2 proteins. The adhesion proteins are the primary method that these cells maintain contact between each other. Eph/ephrin signaling is not well understood but is crucial to the segmentation process in somitogenesis.

In this work the authors do not address the how somite-cycle timing works, they simply use it to describe changes in how cells in different regions express different amounts of proteins. The effects of four proteins are modeled. The four proteins as mentioned before are N-cadherin, N-CAM, EphA4, and ephrinB2. Protein expression does not spatially vary within cells but does vary spatially and temporally within regions of the domain or clusters of cells. There is always a low level of expression of N-CAM and N-caderin for all cells in the domain to keep them loosely connected. The domain is a rectangular region populated with cells. The rectangular region is divided along its length into smaller rectangular regions $S_{i}$ which represent the regions which will form each somite (see the right panel of figure 3). The purpose of the model is to determine if alterations in these four proteins can account for the segmentation process in somitogenesis.

At the beginning of each segmentation cycle, $t_{i}$ for the initiation of somite $i$, the protein levels are set at new levels in the following manner. When a 

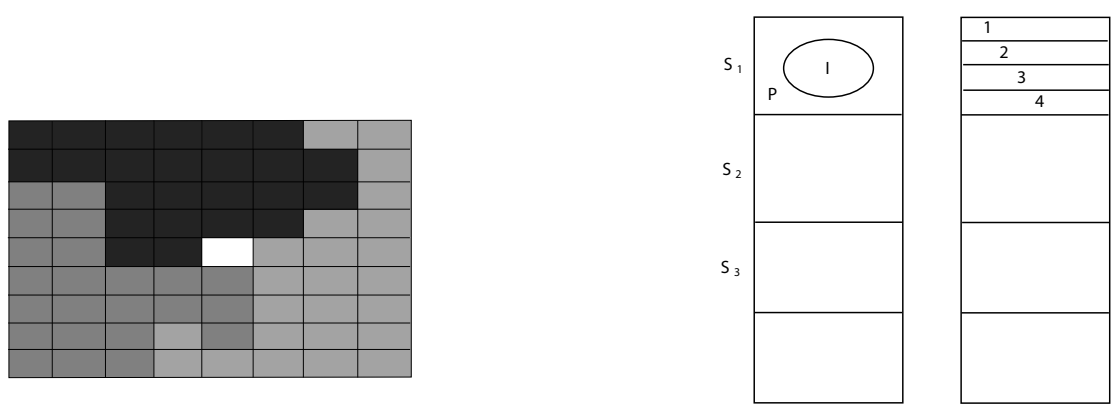

Figure 3: In the left panel a discretized domain for the Cellular Potts Model is shown. There are three cells in the domain; each is indicated by a different shade of gray. The white region is about to be assigned to a cell depending on how the overall energy of the system changes. In the right panel a schematic is shown for a model of somitogenesis. The domain is shown twice and is subdivided into regions $S_{1}, S_{2}$, etc. which will eventually become somites. At the beginning of a segmentation cycle the cells in the periphery (denoted $\mathrm{P}$ in the left domain) have a low expression of N-cadherin and the cells in the interior (I) have a high expression of $\mathrm{N}$-cadherin. At the same time the cells in region 1 of the domain (shown in the right domain) have a high expression of EphA4 and those in region 2 have low expression of EphA4. At the next segmentation cycle the process happens over in $S_{2}$, and in $S_{1}$ the expression of ephrinB2 is low in region 3 and high in region 4. 
segmentation cycle begins, the cells interior to the somite region about to separate from the others have a higher level of expression of N-cadherin and cells at the periphery of the region have a decreased expression of N-cadherin. The front of the region is the region farther away from the unformed somites. The cell in the front quarter region (1) of the forming somite express more EphA4 while those in the second quarter region (2) express low levels of EphA4. At the next segmentation cycle, the levels change in the same way for the next somite region and the ephrinB2 levels in the previous somite region are set to be high in the last quarter region (4) and low in third quarter region (3). This process is repeated with each segmentation cycle.

Initially all the cell sizes are the same, but at the beginning of a segmentation cycle the interior cell sizes are decreased, and the peripheral cell sizes are increased. The extracellular matrix is modeled as a generalized cell.

Cells with high levels of N-cadherin adhere more strongly than those with lower levels to other cells with N-cadherin. Cells with N-CAM adhere to other cells with N-CAM but the adhesion is not as strong as that of N-cadherin. Cell adhesion between cells expressing EphA4 and ephrinB2 is reduced to simulate "cell repulsion". Since the modified Potts method is used, the adhesion and repulsions are modeled as changes in the energy of the cell and the model tries to minimize the total energy.

The authors tried to use only two levels of ephrinB2 and EphA4 (no expression and expression), and although those simulations did result in somite formation, the somites formed an inter somitic notch. At the interface between cells with expression of EphA4 and cells with expression of ephrinB2, the somite would start to form a notch as if it would divide in half. By using two levels of each protein, cells with high ephrinB2 expression, cells with low ephrinB2 expression, cells with high EphA4 expression, and cells with low EphA4 expression the inter somitic notch was much less pronounced or almost disappears. The authors conclude that multiple levels of expression of EphA4 and ephrinB2 are necessary for proper somitogenesis.

Simulations with just one cell adhesion protein also failed to show the proper segmentation of the somites. When there was no difference between N-CAM and N-cadherin, adhesion the somites again divided into two segments at the boundary where cells with low eprhinB2 and low EphA4 are juxtaposed. It is apparently necessary to have a high adhesive core to keep the somite intact. This is consistant with experimental evidence where N-cadherin-double-knockout phenotype show somites which divide into smaller segments [13]

\subsection{Linking the discrete and the continuum}

Deriving equations for continuous variables from basic interactions of discrete objects becomes more difficult as the basic interactions become more complex. Statistical mechanics is used to derive equations for chemical concentrations from the basic interactions of the molecules, for instance deriving the diffusion equation from a random walk. This process has been more difficult for models in biology where the interactions between the discrete objects are

more complex $[17,16]$ and the cells are treated as points. Here we mention the 
work of Alber et. al. who have shown how to go from the Cellular Potts model, an energy conservation based cellular automaton model, for chemotacting cells to the Keller-Segel model, a partial differential equation for chemotacting cells [2]. Their work shows how to take a model which treats cells as extended objects and in the limit arrive at the continuum Keller-Segel equation.

For the one dimensional case the cells are assumed to occupy discrete adjacent lattice sites. The cell can move by occupying or vacating lattices sites. The motion is governed by the Hamiltonian which represents the "energy of the system" and should be minimized. The Hamiltonian for this system is given by

$$
E=J_{c m}\left(2 L+2 \ell_{y}\right)+\lambda\left(L-L_{T}\right)^{2}+\mu c(x) L,
$$

where $L$ is the cell length, and $c(x)$ is the concentration of the chemoattractant. The first term accounts for interaction with the media, the second term penalizes cells if they differ in length from the target length $L_{T}$, and the third term encourages motion due to a chemical gradient.

The master equation for the probability of a cell with center of mass at $x$ and with length $L$ at time $t$ is derived in terms of the transition probabilities of a cell moving from one state to another. The transition probabilities are related to the Hamiltonian and a Fokker-Planck equation is derived. Taking the limit as the distance between lattice sites goes to zero and making various assumptions the probability density can be shown to approach the Boltzmann distribution of the cell's length which is determined. Numerical simulations confirm the analysis. Assuming further that the typical time scale of diffusion is large in comparison with the relaxation time for the Boltzmann distribution the equations for the probability density of a cell's center of mass can be shown to be equivalent to the Keller-Segel model given by

$$
\begin{aligned}
\partial_{t} p & =D \partial_{x}^{2} p-\xi_{0} \partial_{x}\left[p \partial_{x} c\right], \\
\xi_{0} & =\frac{D}{\lambda} \beta \mu\left[J_{c m}-\lambda L_{T}\right] .
\end{aligned}
$$

\section{Conclusions}

The biological sciences is an area where multiscale modeling can play an important role in helping to understand the many important systems. Multiple space and time scales are inherent in these systems and models which account for this can help identify and clarify the mechanisms and key features of each system. There are three trends to take note of in multiscale modeling of biological systems. The first is new innovative models where the system is modeled in detail as Barocas and colleagues have done with collagen tissue [27]. The second trend is to build models of the larger system from models of the individual components as Byrne and colleagues have done with cancerous tissue and angiogenesis [18]. As the smaller components are better understood, it is natural to build models showing how they interact to form the more complex 
system. The final trend is the development of theory to link the discrete multiscale methods to differential equations as Alber and co-workers have done [2]. As these three trends continue helping each other to build better and better models, we will learn to understand, manipulate, and design systems further advancing our biological and medical knowledge. 


\section{References}

[1] T. Alarcon, H. M. Byrne, and P. K. Maini. A cellular automaton model for tumour growth in an inhomogeneous environment. J. Theor. Biol., 225:257-274, 2003.

[2] Mark Alber, Nan Chen, Tilmann Glimm, and Pavel Lushnikov. Twodimensional multiscale model of cell motion in a chemotactic field. In A. R. A. Anderson, M. A. J. Chaplain, and K. A. Rejniak, editors, Singlecell-based models in biology and medicine, pages 53-76. Birkhauser, 2007.

[3] A. R. A. Anderson. A hybid multiscale model of solid tumour growth and invasion: Evolution and the microenvironment. In A. R. A. Anderson, M. A. J. Chaplain, and K. A. Rejniak, editors, Single-cell-based models in biology and medicine, pages 3-28. Birkhauser, 2007.

[4] ${ }^{1}$ A. R. A. Anderson, M. A. J. Chaplain, and K. A. Rejniak, editors. Singlecell-based models in biology and medicine. Birkhauser, 2007.

[5] A. R. A. Anderson, K. A. Rejniak, P. Gerlee, and V. Quaranta. Microenvironment driven invasion: a multiscale multimodel investigation. Journal of Mathematical Biology, 58:579-624, 2009.

[6] R. P. Araujo and D. L. S. McEwain. A history of the study of solid tumour growth: the contribution of mathematical modeling. Bull. Math. Biol., 66:1039-1091, 2004.

[7] Preethi L. Chandran, Triantafyllos Stylianopoulos, and Victor H. Barocas. Microstructure-based, multiscale modeling for the mechanical behavior of hydrated fiber networks. Multiscale model Simul., 7(1):22-43, 2008.

[8] N. Chen, J. A. Glazier, J. A. Izaguirre, and M. S. Alber. A parallel implementation of the cellular potts model for simulation of cell-based morphogenesis. Computer Physics Communications, 176:670-681, 2007.

[9] J. C. Dallon, E. Newren, and M. D. H. Hansen. Using a mathematical model of cadherin-based adhesion to understand the function of the actin cytoskeleton. Physical Review E, 79(3):031918, 2009.

[10] J. C. Dallon, J. A. Sherratt, and Philip K. Maini. Mathematical modelling of extracellular matrix dynamics using discrete cells: Fiber orientation and tissue regeneration. J. Theor. Biol., 199(4):449-471, Aug 1999.

[11] J. A. Glazier, Y. Zhang, M. Swat, B. Zaitlen, and S. Schnell. Coordinated action of n-cam, n-cadherin, epha4, and ephrinb2 translates genetic prepatterns into structure during somitogenesis in chick. In S. Schnell, P. K. Maini, S. A. Newman, and T. J. Newman, editors, Multiscale Modeling of

\footnotetext{
${ }^{1}$ Compilation of several multiscale models in biology
} 
Developmental Systems, volume 81 of Current Topics in Developmental Biology, pages 205-247. Elsevier, 2008.

[12] R. Haggkvist, A. Rosengren, P. H. Lundow, K. Markstrom, D. Andren, and P. Kundrotas. On the ising model for the simple cubic lattice. Advances in Physics, 55(5):653-755, 2007.

[13] K Horikawa, G. Radice, M. Takeichi, and O. Chisaka. Adhesive subdivisions intrinsic to the epithelial somites. Dev. Biol., 215:182-189, 1999.

[14] Xiao-Juan Luo, Triantafyllos Stylianopoulos, Victor H. Barocas, and Mark S. Shephard. Multiscale computation for bioartificial soft tissues with complex geometries. Engineering with Computers, 25:87-95, 2009.

[15] S. McDougall, J. Dallon, J. Sherratt, and P. Maini. Fibroblast migration and collagen deposition during dermal wound healing: mathematical modelling and clinical implications. Phil. Trans. R. Soc. London, Ser. A, 364:1385-1405, 2006.

[16] T. J. Newman and R. Grima. The many-body theory of chemotactic cellcell interactions. Phys. Rev. E, 70:051916:1-15, 2004.

[17] Hans G. Othmer and A. Stevens. Aggregation, blowup, and collapse: The abcs of taxis in reinforced random walks. SIAM J. Appl. Math, 57(4):10441081, 1997.

[18] M. R. Owen, T. Alarcon, P. K. Maini, and H. M. Byrne. Angiogenesis and vascular remodelling in normal and cancerous tissues. J. Math. Biol., 58:689-721, 2009.

[19] S. Pennacchietti, P. Michieli, M. Galluzzo, M. Mazzone, S. Giordano, and P. M. Comoglio. Hypoxia promote invasive growth by transcriptional ativation of the met protooncogene. Cancer Cell, 3:347-361, 2003.

[20] C. S. Peskin. Flow patterns around heart valves: A numerical method. J. Comput. Phys., 10:252-271, 1972.

[21] C. S. Peskin. Teh immersed boundary method. Acta Numerica, 11:479-517, 2002.

[22] K. A. Rejniak. Modelling the development of complex tissues using individual viscoelastic cells. In A. R. A. Anderson, M. A. J. Chaplain, and K. A. Rejniak, editors, Single-cell-based models in biology and medicine, pages 301-323. Birkhauser, 2007.

[23] K. A. Rejniak and A. R. A. Anderson. A computational study of the development of epithelial acini: I sufficient conditions for the formation of a hollow structure. Bulletin of Mathematical Biology, 70:677-712, 2008. 
[24] E. A. Sander, T. Stylianopoulos, R. T. Tranquillo, and V. H. Barocas. Image-based biomechanics of collagen-based tissue equivalents: multiscale model compared to fiber alignment predicted by polarimetric imaging. IEEE Engineering in Medicine and Biology, 2009.

[25] ${ }^{1}$ S. Schnell, P. K. Maini, S. A. Newman, and Newman T. J., editors. Mutliscale Modeling of Developmental Systems, volume 81 of Current Topics in Developmental Biology. Elsevier, 2008.

[26] Triantafyllos Stylianopoulos and Victor H. Barocas. Multiscale, structurebased modeling for the elastic mechanical behavior of arterial walls. Journal of Biomechanical Engineering, 129:611-618, 2007.

[27] Triantafyllos Stylianopoulos and Victor H. Barocas. Volume-averaging theory for the study of the mechanics of collagen networks. Comput. Methods Appl. Mech. Engrg., 197:2981-2990, 2007.

[28] J. J. Tyson and Novaka B. Regulation of te eukaryotic cell-cycle: molecular anatagonism, hysteresis, and irreversible transitions. J. Theor. Biol., 210:249-263, 2001. 